\title{
Erratum to: Multivariate spectral multipliers for tensor product orthogonal expansions
}

\author{
Błażej Wróbel
}

Published online: 25 November 2012

(C) Springer-Verlag Wien 2012

\section{Erratum to: Monatsh Math (2012) 168:125-149 DOI 10.1007/s00605-011-0363-8}

In order for the main theorem of the original paper to be true one needs the additional assumption on $L^{p}$ contractivity of the heat semigroups of the investigated operators. We need to assume that for each $n=1, \ldots, d$ and all $p \in[1, \infty]$,

$$
\left\|e^{-t_{n} \mathcal{L}_{n}} f\right\|_{L^{p}\left(A_{n}, v_{n}\right)} \leq\|f\|_{L^{p}\left(A_{n}, v_{n}\right)}, \quad t_{n}>0, \quad f \in L^{p}\left(A_{n}, v_{n}\right) \cap L^{2}\left(A_{n}, v_{n}\right)
$$

Since (1) implies item (ii) of Theorem 2.3, to draw the conclusion of the main theorem (Theorem 2.3) of the original paper (i.e. the $L^{p}$ boundedness of the operator $m(\mathcal{L})$ ), it now suffices to assume only item (i),

$$
\int_{\mathbb{R}^{d}} \sup _{T \in(0, \infty)^{d}}\left|\mathcal{M}\left(m_{N, T}\right)\left(u_{1}, \ldots, u_{d}\right)\right|\left\|\mathcal{L}^{i u_{1}, \ldots, i u_{d}}\right\|_{p \rightarrow p} d u<\infty
$$

We kindly refer the reader to the original paper for the definitions of the quantities considered above. Condition (1) is not a serious restriction and it is satisfied in case of all applications presented in the original paper. We need to include (1) in order to obtain Theorem 2.4 for $1<p<2$, i.e. the $L^{p}$ boundedness of the $g$-function $g_{N}$

The online version of the original article can be found under doi:10.1007/s00605-011-0363-8.

B. Wróbel (凶)

Institute of Mathematics of University of Wrocław, pl. Grunwaldzki 2/4, 50-384 Wrocław, Poland e-mail: blazej.wrobel@math.uni.wroc.pl 
for $1<p<2$. The proof of Theorem 2.4 from the original paper, is incorrect for $1<p<2$. The correction we present here is a slight modification of the proof of [2, Theorem $1.5 \mathrm{ii})]$.

For the sake of simplicity we focus on $d=2$. The notations we use are from the original paper. Let $N \in \mathbb{N}_{+}$be fixed. Take a smooth function $h$ on $\mathbb{R}$, supported in $[-1,1]$, and such that

$$
\sum_{l \in \mathbb{Z}} h(x-l)=1, \quad x \in \mathbb{R}
$$

Then we set $h_{k}(x)=h_{k_{1}}\left(x_{1}\right) h_{k_{2}}\left(x_{2}\right)=h\left(x_{1}-k_{1}\right) h\left(x_{2}-k_{2}\right)$. Next, for each $j \in \mathbb{Z}^{2}$ we define the functions

$$
\begin{aligned}
b_{j, k}(\xi)= & \int_{\mathbb{R}^{2}} h_{k}(u) \Gamma\left(N-i u_{1}\right) \Gamma\left(N-i u_{2}\right) e^{-i j_{1} u_{1}} e^{-i j_{2} u_{2}} \xi_{1}^{i u_{1}} \xi_{2}^{i u_{2}} d u \\
= & \int_{\mathbb{R}} h_{k_{1}}\left(u_{1}\right) \Gamma\left(N-i u_{1}\right) e^{-i j_{1} u_{1}} \xi_{1}^{i u_{1}} d u_{1} \\
& \times \int_{\mathbb{R}} h_{k_{2}}\left(u_{2}\right) \Gamma\left(N-i u_{2}\right) e^{-i j_{2} u_{2}} \xi_{2}^{i u_{2}} d u_{2}, \quad \xi_{1}, \xi_{2} \in \Sigma_{\pi / 2},
\end{aligned}
$$

where $\Sigma_{\pi / 2}=\{z \in \mathbb{C}: \operatorname{Re}(z)>0\}$, is the right complex half plane. Proceeding as in $[2$, p. 2207] we easily see that

$$
\left\|g_{N}(f)\right\|_{p} \leq \sum_{k \in \mathbb{Z}^{2}}\left\|\left(\sum_{j \in \mathbb{Z}^{2}}\left|b_{j, k}\left(L_{1}, L_{2}\right) f\right|^{2}\right)^{1 / 2}\right\|_{p} .
$$

Then from a two-dimensional variant of [2, Lemma 1.3] (which is easily proved by using a two-dimensional Khinchine inequality) and the product structure of $b_{j, k}$ it follows that

$$
\left\|g_{N}(f)\right\|_{p} \leq \sum_{k \in \mathbb{Z}^{2}} \sup _{\left|a_{j_{1}}\right| \leq 1,\left|a_{j_{2}}\right| \leq 1}\left\|\left(\sum_{j_{1} \in \mathbb{Z}} a_{j_{1}} b_{j_{1}, k_{1}}\left(L_{1}\right)\right)\left(\sum_{j_{2} \in \mathbb{Z}} a_{j_{2}} b_{j_{2}, k_{2}}\left(L_{2}\right)\right) f\right\|_{p} .
$$

Using the latter inequality and [2, Lemma 1.4] we easily adjust the final steps of [2, Theorem 1.5 ii)] to our situation, obtaining the desired bound

$$
\left\|g_{N}(f)\right\|_{p} \leq C_{p}\|f\|_{p}, \quad 1<p<2 .
$$

Note that assumption (1) is needed to justify the use of the crucial transference result of [1, Theorem 1 and Lemma 1.4]. 


\section{References}

1. Cowling, M.G.: Harmonic analysis on semigroups. Ann. Math. 117, 267-283 (1983)

2. Meda, S.: On the Littlewood-Paley-Stein g-function. Trans. Am. Math. Soc. (3) 110, 639-647 (1990) 